\title{
6. Rezensionsabhandlungen
}

Johannes Rux: Direkte Demokratie in Deutschland. Rechtsgrundlagen und Rechtswirklichkeit der unmittelbaren Demokratie in der Bundesrepublik Deutschland und ihren Ländern. Nomos Verlagsgesellschaft: Baden-Baden 2008, 984 S., 158 Euro.

Die direkte Demokratie hat in Deutschland Konjunktur. So brachte in Hamburg jüngst ein aufsehenerregender Volksentscheid vom Juli 2010 die Pläne des dortigen Senats aus CDU und Grünen zum Scheitern, eine sechsjährige gemeinsame Primarschule für alle schulpflichtigen Kinder einzurichten. Die im Landesverfassungsrecht in vielfältigen Formen vorgesehenen direktdemokratischen Verfahren, die jahrzehntelang kaum eine Rolle gespielt hatten, werden seit Beginn der neunziger Jahre häufiger genutzt. Dies könnte mit dem gelegentlich als „Glokalisierung" bezeichneten Phänomen zusammenhängen, dass die Bürger unter dem Eindruck der zunehmenden Verlagerung von Entscheidungskompetenzen auf überstaatliche Ebenen gewissermaßen kompensatorisch alle Möglichkeiten ausschöpfen, ihr unmittelbares Lebensumfeld selbst zu gestalten. Jenseits der Länderebene wird immer wieder diskutiert, ob entsprechende Verfahren auch im Bund eingerichtet werden sollten, wo das Grundgesetz bekanntlich Volksabstimmungen derzeit nur nach Art. 29 GG für den Fall von Länderneugliederungen vorsieht. Die Tübinger Habilitationsschrift von Johannes Rux kommt also zur rechten Zeit. Sie bietet eine umfassende Bestandsaufnahme der direkten Demokratie in der Bundesrepublik. Verdienstvollerweise behandelt Verf. dabei nicht allein die durch die unmittelbare Demokratie aufgeworfenen Rechtsfragen, sondern stellt auch eingehend die bisherige Praxis dar.

Verf. wählt im Kern einen historischen Aufbau. Der erste Teil soll in das Sachproblem einführen. Danach folgen drei Teile zur Geschichte der direkten Demokratie in Deutschland vor 1945, zur Entwicklung in Westdeutschland zwischen 1945 und 1989 und schließlich zu Regelungen und Praxis in den neueren Landesverfassungen von der Wiedervereinigung bis heute. Ein fünfter abschließender Teil bewertet das geltende Recht zusammenfassend und formuliert Vorschläge für eine zukünftige Weiterentwicklung der direkten Demokratie in Deutschland. Den Löwenanteil des Buches nimmt dabei die detaillierte Darstellung der Verfahren in den Landesverfassungen ein, die seit der Wiedervereinigung verabschiedet oder grundlegend revidiert wurden (Schleswig-Holstein, Brandenburg, Sachsen, Sachsen-Anhalt, Niedersachsen, Mecklenburg-Vorpommern, Thüringen, Bremen, Berlin, Hamburg, Rheinland-Pfalz, Nordrhein-Westfalen). 
Als direktdemokratisch versteht Verf. alle Verfahren, die innerhalb des Staates zu einer unmittelbaren Sach- oder Personalentscheidung durch das Staatsvolk führen (S. 38). Diese Definition weicht vom herkömmlichen Sprachgebrauch insofern ab, als sie auch die Parlamentswahl als direktdemokratisch begreift (S. 39). Nun sind Definitionen stets in weitem Umfang eine Frage der Konvention. Auch in der Parlamentswahl geht es in der Tat um Personalentscheidungen durch das Staatsvolk; insoweit besteht durchaus eine Parallele etwa zur Direktwahl des Staatsoberhaupts, wie es sie beispielsweise in Frankreich oder Österreich gibt. Freilich sind die Sach- und Rechtsprobleme, die unmittelbare Sachentscheidungen durch das Staatsvolk aufwerfen, in vielem anders gelagert als Fragen der Direktwahl von Staatsorganen. Verf. bringt die Parlamentswahl in diesem Zusammenhang vor allem deshalb ins Spiel, weil sie ihm Gelegenheit bietet, ,die strukturellen Probleme der repräsentativ-parlamentarischen Demokratie“ zu erörtern (S. 69 ff.), und widmet seine Studie ansonsten insgesamt nicht den Parlamentswahlen, sondern den Sachentscheidungen durch das Volk.

Die Möglichkeiten und Aufgaben direktdemokratischer Mitwirkungsformen erschließt die Studie allgemein dadurch, dass sie auf „strukturelle Probleme“ der parlamentarischen Demokratie verweist (S. 69 ff.). In einem stark von Parteien dominierten repräsentativen System sei ,nicht hinreichend sicher gestellt, dass sich die Parteien und die auf ihren Vorschlag gewählten Amts- und Mandatsträger am Gemeinwohl oder zumindest an dem orientieren werden, was eine hinreichende Mehrheit der Bürger für das Gemeinwohl hält"“ (S. 70). Dieser Kritikhorizont ist freilich nicht unproblematisch, kann sich das Gemeinwohl doch ohnehin erst als Resultante der entsprechenden öffentlichen und institutionellen Willensbildungsprozesse herausbilden. Ebenso fragwürdig ist die Behauptung, die Gewaltenteilung werde ,durch den dominierenden Einfluss der Parteien überspielt“ (S. 81). Es ist dem parlamentarischen Regierungssystem in Bund und Ländern vielmehr bereits durch die vorgeschriebene Abhängigkeit der Regierung vom Vertrauen des Parlaments immanent, dass Parlamentsmehrheit und Regierung hier in einer engen Symbiose stehen. Eine Gewaltenteilung im US-amerikanischen Sinne, die erste und zweite Gewalt strikt trennt, kennen weder das Grundgesetz noch die Landesverfassungen. Manches deutet sicherlich darauf hin, dass sich in Deutschland eine wachsende Entfremdung der Bürger von den Institutionen abzeichnet (S. 82). Die Zurechnung dieses Problembefunds zum parlamentarischen Regierungssystem und den Parteien ist aber zu einfach.

Bei allen Schwierigkeiten der Problemdiagnose wird man Verf. aber zustimmen können, dass direktdemokratische Verfahren durchaus eine komplementäre Funktion zum repräsentativen Parlamentarismus haben können (S. 83 ff.). Es versteht sich, dass der Autor einer Habilitationsschrift über direkte Demokratie 
dieser auch etwas abgewinnen kann. Verf. ist aber kein Zelot der unmittelbaren Demokratie und verfällt auch nicht auf die in einem Strang der deutschen Tradition politischen Denkens verbreitete Idealisierung der vermeintlich „,herrschaftsfreien“ direkten Demokratie. Er wendet sich aber gegen die in der Bundesrepublik lange Zeit vorherrschende Grundsatzkritik an direktdemokratischen Mechanismen aus der Perspektive eines strikt repräsentativen Parlamentarismus (S. 91 ff.). Bei jenen Beteiligungsformen gehe es vielmehr darum, die Kommunikation zwischen Bürgern und Repräsentanten zu verbessern und den öffentlichen Diskurs über Sachfragen zu institutionalisieren und zu kanalisieren (S. 84). Die Einwände der Gegner (fehlende Kompetenz der Bürger, „Diktatur der Minderheit“, Anfälligkeit für Demagogie, höhere Qualität der Ergebnisse parlamentarischer Prozesse, mögliche destabilisierende Wirkung) werden im Einzelnen erörtert und kritisiert.

Verf. hat recht, wenn er insoweit für eine stärkere Versachlichung der Diskussion plädiert. Die Debatte in der Bundesrepublik war sicherlich über Jahrzehnte von einer spezifischen Stabilitätssehnsucht geprägt, die allem Plebiszitären mit Misstrauen begegnete. Hierbei wirkte lange das schon im Parlamentarischen Rat wichtige Argument nach, die Plebiszite der Weimarer Zeit, insbesondere das Volksbegehren gegen den Young-Plan (dazu S. 153 ff.), hätten der Demagogie einen besonderen Raum verschafft und damit nicht unerheblich zum Untergang der Republik beigetragen. Die historische Forschung zur Weimarer Republik, die Verf. rezipiert (S. 108 ff., bes. 191 f.), ist hier inzwischen zu einer deutlich vorsichtigeren Einschätzung gelangt. Die zeitgenössischen Plebiszite haben die Weimarer Republik zwar nicht stabilisiert, aber auch nicht maßgeblich zu ihrer aus anderen Gründen großen Instabilität beigetragen. Für die Skepsis des Parlamentarischen Rats gegenüber Volksbegehren und Volksentscheid waren insoweit keineswegs allein Weimarer Eindrücke der Verfassungsväter maßgeblich; es spielte dabei zudem die zeitgenössische Furcht vor einer Instrumentalisierung derartiger Verfahren durch die KPD eine Rolle (dazu S. 206 ff.). Gerade vor diesem Hintergrund hätte die systematische Argumentation der Studie gewonnen, wenn Verf. stärker im Zusammenhang auf heutige ausländische Erfahrungen mit der direkten Demokratie, etwa in der Schweiz und den Vereinigten Staaten, eingegangen wäre. Verf. berücksichtigt zwar gelegentlich die diesbezügliche Sekundärliteratur, wendet sich aber gegen entsprechende vergleichende Argumente, weil das jeweilige Institutionengefüge zu unterschiedlich sei (S. 65 ff.). So richtig es ist, dass derartige ,legal transplants“ immer einer besonders sorgfältigen Kontextualisierung bedürfen, so sehr hätte doch ein zusammenfassender Blick auf die alltägliche Praxis direkter Demokratie in den Kantonen der Schweiz und den Bundesstaaten der USA die Gesamtargumentation bereichert 
und verhindert, dass diese manchmal zu schnell auf eine allzu technische Ebene gerät.

Im Rahmen des ersten Teils scheint dabei bereits eines der Grundsatzprobleme direktdemokratischer Verfahren auf, das insbesondere im Zusammenhang mit der Abschaffung des bayerischen Senats durch verfassungsändernden Volksentscheid im Jahr 1998 diskutiert worden ist: die Frage nach erforderlichen Mehrheiten und Abstimmungsquoren (S. 51 ff.). Verf. wendet sich hier gegen Beteiligungsquoren, weil er die Auswirkung der Nichtbeteiligung eines Stimmberechtigten an der Abstimmung völlig neutral halten will. Nichtbeteiligung sei gleichbedeutend mit Indifferenz. Das Fernbleiben eines Stimmberechtigten von der Abstimmung dürfe sich daher weder für noch gegen den zur Abstimmung gestellten Vorschlag auswirken. Für den Erfolg in der Abstimmung fordert er dann die absolute Mehrheit der abgegebenen gültigen Stimmen (S. 54 f.; vgl. auch S. 249 f.). Das ist selbstverständlich eine vertretbare Position. Aber sie wird nicht intensiver diskutiert und erläutert. Da es sich um ein zentrales Problem handelt, hätte die Studie es an irgendeiner Stelle umfassender behandeln müssen. Dabei wäre auch genauer zu erörtern gewesen, inwieweit sich die unmittelbare Volksgesetzgebung von den parlamentarischen Abstimmungsregeln unterscheidet. Verf. kontrastiert beide Fälle kursorisch: Im Parlament hätten die Abgeordneten nicht nur das Recht, sondern auch die Pflicht, Entscheidungen anstelle der Bürger zu treffen. Für Stimmenthaltung bleibe hier grundsätzlich kein Raum, Mehrheit der Mitglieder sei konsequenterweise als Mehrheit der gesetzlichen Mitgliederzahl - wie in der Definition des Art. $121 \mathrm{GG}$ - zu verstehen (S. 54 mit Fn. 2). Anders lägen die Dinge bei der unmittelbaren Volksgesetzgebung, bei der Stimmenthaltung grundsätzlich möglich sein und im Hinblick auf die Mehrheitsbildung folgenlos bleiben müsse. Diese Kontrastierung überzeugt so aber nicht. Betrachtet man nur einmal die parlamentarischen Abstimmungsregeln nach dem Grundgesetz, so ist dort für einen Gesetzesbeschluss regelmäßig gerade nicht die Mehrheit der gesetzlichen Mitgliederzahl im Sinne von Art. 121 GG erforderlich, sondern lediglich die Mehrheit der abgegebenen Stimmen (Art. 42 Abs. 2 Satz 1 GG). Deshalb wird bekanntlich darüber diskutiert, ob aus dem Demokratieprinzip des Grundgesetzes Mindestquoren für die Beschlussfähigkeit des Bundestages abzuleiten sind. Vor diesem Hintergrund wäre es sinnvoll gewesen, die Regeln über Beteiligungsquoren und Mehrheiten bei der parlamentarischen Gesetzgebung und der unmittelbaren Volksgesetzgebung näher systematisch gegenüberzustellen, so dass die jeweiligen Eigenheiten klarer hätten hervortreten können.

Die gewählte Darstellungsform hat insgesamt den Vorteil, dass eine Entwicklungsgeschichte deutlich wird. Die jeweiligen Regelungen knüpfen häufig an 
Vorgängernormen seit der Weimarer Republik an oder reagieren jedenfalls auf die älteren Regeln. Der deutsche historische Erfahrungsraum mit direkter Demokratie seit dem Ende der Monarchie vor gut neunzig Jahren wird so als ganzer kenntlich. Zudem werden dem Leser die jeweiligen verfassungsrechtlichen Regeln und praktischen Anwendungsbeispiele in enzyklopädischer Breite vorgeführt. Es liegt hier ein in mühseliger Kleinarbeit geschaffenes, sehr verdienstvolles Nachschlagewerk vor.

Diese Vorteile werden allerdings mit einigen Nachteilen erkauft. Der Leser stößt immer wieder auf allgemeine Strukturprobleme, die in allen untersuchten Verfassungen in wechselnden Formen auftauchen: etwa die bereits angesprochene Problematik der erforderlichen Quoren, die Verzahnung von Volksentscheid und Volksbegehren mit den jeweiligen Parlamenten oder der Ausschluss bestimmter Sachfragen wie Abgaben und Haushalt aus dem Anwendungsbereich der Volksgesetzgebung. Verf. erörtert diese Fragen aber, von einzelnen Ausführungen im Einleitungskapitel abgesehen, an keiner Stelle systematisch; sie tauchen vielmehr über die Arbeit verstreut immer wieder auf. Man kann das etwa an dem wichtigen Beispiel sehen, inwieweit Finanz- und Haushaltsfragen einem Volksentscheid unterliegen können. Verf. behandelt das Sachproblem und die Gründe für einen entsprechenden Ausschluss nicht gebündelt. Es taucht aufgrund der historischen Darstellungsweise vielmehr zunächst in den Ausführungen zur Weimarer Situation auf (S. 119 ff.). Verf. wundert sich dort mit Recht darüber, mit welcher Selbstverständlichkeit Finanzfragen damals aus dem Volksentscheid ausgeklammert wurden - mit prägenden Nachwirkungen bis heute -, obwohl es im naheliegenden Schweizer Vergleichsfall für Abgabengesetze keine derartigen Beschränkungen gab. Er stellt die plausible Vermutung auf, dass die deutschen Parlamentarier die Finanzhoheit auch deshalb nicht preisgeben wollten, weil die Volksvertretungen das Budgetrecht im Rahmen der konstitutionellen Monarchie erst wenige Jahrzehnte früher endgültig erkämpft hatten. Hier zeigt sich ein deutlicher Unterschied zur Schweizer Situation, wo die direktdemokratischen Verfahren dem liberalen Bürgertum, das den Berner Bundesrat nach 1848 jahrzehntelang dominierte, in der zweiten Hälfte des 19. Jahrhunderts durch die demokratische Bewegung zunächst katholischer und dann sozialdemokratischer Provenienz abgetrotzt worden waren (S. 120 mit Fn. 2). Derartige instruktive Einzelbeobachtungen werden aber nicht zum Anlass genommen, eine systematische Fragestellung zu entwickeln. Stattdessen begegnet das Thema verstreut über die Arbeit immer wieder etwa in Ausführungen zu den Anforderungen von Art. 28 Abs. $1 \mathrm{GG}$ an die Landesverfassungen (S. 243 ff.) und zu einzelnen landesrechtlichen Bestimmungen (etwa S. 271 f., 273 ff., 638 ff.). Der gewählte historischenzyklopädische Aufbau führt dazu, dass die zugrundeliegende Sachfrage an 
keiner Stelle konzentriert behandelt wird und bedenkenswerte systematische Argumente des Verfassers - etwa die kritische Auseinandersetzung mit der extensiven Rechtsprechung des Bayerischen Verfassungsgerichtshofs zum Haushaltsvorbehalt der bayerischen Verfassung (S. 273 ff.) - nicht zusammenhängend dargestellt werden.

Die Gesamtbilanz des Verf. zu den bisherigen Erfahrungen mit direkter Demokratie in der Bundesrepublik (S. 904 ff.) fällt ernüchternd aus. Verf. macht dafür vor allem die vergleichsweise hohen Quoren für Volksbegehren und Volksentscheid im geltenden Landesrecht verantwortlich. Hinzu kommen allerdings wohl andere Aspekte, die viel schwerer änderbar sind. Zu ihnen zählt aus Sicht des Rezensenten die unitarische Struktur des deutschen Bundesstaats. Es fehlt hier jenes ausgedehnte Feld der Landesgesetzgebung, das etwa in der Schweiz und den USA der natürliche Betätigungsbereich der direkten Demokratie ist. Fehlt es aber an verantwortlicher Einübung der Volksgesetzgebung in den Ländern, dann hinge diese auch im Bund gewissermaßen in der Luft. Die starke Einschnürung durch verfassungsrechtliche Vorgaben kommt noch erschwerend hinzu. Rechtspolitisch macht Bearb. überdies darauf aufmerksam, dass in den Ländern bisher vor allem solche Anträge Erfolg hatten, die sich gegen Reformvorhaben der jeweiligen Regierungsmehrheit richteten. Die direkte Demokratie begründet häufig zusätzliche Vetopositionen und wirkt tendenziell auf die Erhaltung des status quo hin, wie auch die Schweizer Erfahrungen zeigen. Verf. verweist aber auch auf andere Beispiele, in denen Volksinitiativen Reformen erst anstießen, und fordert in jedem Fall rechtspolitisch die Absenkung der Quoren und eine Erweiterung des Anwendungsbereichs direktdemokratischer Verfahren bzw. deren Einführung auch auf Bundesebene. Es spricht für die wissenschaftliche Redlichkeit des Verf., dass er in seinen eigenen Forderungen ein ungewisses „Wagnis“ sieht (S. 930).

Ungeachtet mancher Einzelkritik liegt hier eine tiefgehend dokumentierte Gesamtstudie vor, die ein umfassendes Bild der Situation der direkten Demokratie in Deutschland erschließt. Direktdemokratische Verfahren werden ihren Platz hier auf Dauer nur festigen können, wenn sie auf ihre Weise zur Erneuerung und Anpassungsfähigkeit des repräsentativen Parlamentarismus beitragen.

Christoph Schönberger

Kenneth P. Miller: Direct Democracy and the Courts, Cambridge University Press, Cambridge u.a. 2009, VIII, 278 S., 50,- £ bzw. 16,99 £ (Gebundene Ausgabe/Taschenbuch). 
Direktdemokratische Initiativen scheitern in Deutschland - zumindest auf Landesebene - mehrheitlich nicht im Volksentscheid, sondern schon im Vorfeld desselben vor Gericht. Das Verhältnis von unmittelbarer Demokratie und (Verfassungs-)Gerichtsbarkeit darf vor diesem Hintergrund durchaus als spannungsreich umschrieben werden.

Erwartungsvoll nimmt der deutsche Leser daher die Studie des am Claremont McKenna College (Kalifornien) lehrenden Rechts- und Politikwissenschaftlers Kenneth P. Miller zur Hand, der sich dem Verhältnis, präziser den teils heftigen Auseinandersetzungen von direkter Demokratie und Gerichtsbarkeit in den Vereinigten Staaten widmet. Er wird - soviel vorweg - nicht enttäuscht, sondern erhält einen kenntnisreichen und subtil abwägenden Überblick, der teils frappierende Parallelen zur deutschen Situation aufweist, aber auch deutlich werden läßt, wo die Grenze der Vergleichbarkeit der beiden Systeme verläuft. Einziges Manko ist vielleicht, daß der Band zwar dem mit der deutschen Debatte vertrauten Leser einen solchen Vergleich ermöglicht, diesen aber selbst nicht anstellt (bzw. bis auf wenige Ausnahmen auf grenzüberschreitende und rechtsvergleichende Hinweise gänzlich verzichtet).

Ausgangspunkt und im Verlauf der Untersuchung immer wieder aufgenommener roter Faden ist die - sich praktisch vor den Augen des Verfassers abspielende - Auseinandersetzung um die gleichgeschlechtliche Ehe in Kalifornien (S. 3-13): Auf die einfachgesetzliche Festlegung, daß eine Ehe nur zwischen Mann und Frau geschlossen werden könne (Proposition 22, 2000), folgte hier 2008 die Entscheidung des obersten Gerichts des Bundesstaates, daß dieses Gesetz den Gleichheitssatz der kalifornischen Verfassung verletze, diese m.a.W. einen Anspruch Homosexueller auf Eheschließung enthalte. Die Gegner der „Homoehe“ starteten daraufhin eine Verfassungsinitiative, welche die in Proposition 22 enthaltene Regelung nunmehr in die kalifornische Verfassung aufnehmen sollte; diese Proposition 8 hatte am Tag der Wahl Barack Obamas zum Präsidenten Erfolg. Millers Prognose, daß Proposition 8 anschließend vor Bundesgerichten angefochten werden und dort zumindest eine teilweise Niederlage erleiden würde, hat sich mittlerweile bestätigt: Unlängst hat ein erstinstanzliches Bundesgericht den Ausschluß der gleichgeschlechtlichen Ehe als Verstoß gegen die Bundesverfassung gewertet. Die endgültige Entscheidung dieser intensiv debattierten gesellschaftspolitischen Frage steht noch aus; daß sie letztlich allein in den Händen der Richterinnen und Richter des US Supreme Court (und nicht in denen eines Parlaments) liegt, beunruhigt nicht nur Miller.

Dieser verschafft dem Leser zunächst einen konzisen historischen Überblick (S. 17-40): Während die vielzitierten „Gründerväter“ der direkten Demokratie aus Angst vor einer Tyrannei der Mehrheit und in Sorge um die Möglichkeit, das 
Plebiszit in das System der ,,checks and balances“ zu integrieren, zutiefst skeptisch gegenüberstanden, erfolgte die Einführung der verschiedenen Instrumente der unmittelbaren Demokratie im Kern in den Jahren 1898 bis 1918 und vorwiegend in westlichen Bundesstaaten. Erfolg hatte diese „progressive“ Bewegung unter dem Eindruck von Cliquenwirtschaft und Korruption im politischen System; sie setzte allerdings laut Miller die Initiative in ihren verschiedenen Spielarten unverbunden neben das Repräsentativsystem, ohne an eine Koordinierung beider zu denken. Zum Problem wird dies erst, so der nächste gut nachvollziehbar geschilderte Schritt (S. 41-71), als die direkte Demokratie nach einer ersten Phase der Hochkonjunktur (1900-1910) und einem längeren Moratorium (19201970) ab den Siebziger Jahren wieder an Fahrt gewinnt und in einigen Staaten (an erster Stelle Kalifornien) die Form einer ,initiative industry“ (S. 53) annimmt. Miller schildert materialreich und mit viel Übersicht, aber unter Verzicht auf jede Form von Datenhuberei die tatsächliche Entwicklung und benennt die Schwerpunkte des Einsatzes direktdemokratischer Instrumente.

Ausgehend von der offensichtlichen Möglichkeit, daß (direkt-)demokratische Mehrheiten Entscheidungen fällen, die entweder gemeinwohlwidrig sind oder Minderheiten benachteiligen, fragt Miller weiter nach den Möglichkeiten der übrigen Verfassungsorgane, solche Entscheidungen zu korrigieren (S. 75-100). Daß er Exekutive wie Legislative für praktisch machtlos hält und allein die Gerichte in der Lage sieht, Fehlentwicklungen bzw. Fehlentscheidungen zu begegnen, akzentuiert den wohl wichtigsten Unterschied zwischen der deutschen und USamerikanischen Rechtsordnung in Sachen „Direkte Demokratie und Gerichtsbarkeit“: Während in der Bundesrepublik tief gestaffelte Programme der präventiven Kontrolle direktdemokratischer Instrumente existieren, die Parlament, Regierung und - im Sinne einer Letztentscheidung - die Verfassungsgerichte involvieren, laufen Initiativen in den Gliedstaaten der USA - mit wenigen Ausnahmen am Repräsentativsystem vollständig vorbei und gelangen erst dann vor Gericht, wenn das Volk seine Stimme bereits abgegeben hat - was einer etwaigen gerichtlichen Kassation des Volkswillens ein ganz anderes Gewicht gibt und gehässigen Wortspielen Vorschub leistet, sieben, acht oder neun Richter hätten den Willen von soundso viel Millionen Bürgern ignoriert.

Die sich anschließende Detailuntersuchung der Rechtsprechung zur direkten Demokratie (S. 101-123) weckt beim deutschen Leser immer wieder Assoziationen, wenn etwa in der Verfassung vorgesehene rein formale Kriterien von den Gerichten freihändig inhaltlich aufgeladen werden oder ein südöstlicher Bundesstaat durch eine überkritische Auslegung der ,single subject rule“ auffällt (S. 121 f.; zu deutsch resp. bayerisch: Koppelungsverbot). Miller unterscheidet im folgenden nach Konflikten über (Grund-)Rechte (S. 124-155) und Kompetenzen (S. 
156-185). Er geht dabei sehr skrupulös der Frage nach, ob und in welchem Umfang direkte Demokratie eine Gefahr für die Grundrechte von Minderheiten darstellt; sein Ergebnis, daß direktdemokratische Initiativen in der Tat die Ausdehnung von Individualrechten verlangsamt haben, dieser Effekt von der grundrechtsfreundlichen, ja -enthusiastischen Rechtsprechung aber mehr als kompensiert worden sei, überzeugt angesichts der präsentierten Fakten. Tendenziell kritischer bewertet er die direktdemokratisch induzierten Veränderungen im Bereich der Staatsorganisation; hier sei die „Gegenmacht“ der Gerichte zumal dann begrenzt, wenn sie allein auf der Grundlage der Verfassung des Einzelstaats zu entscheiden hätten, die ihrerseits dem Zugriff des Volksgesetzgebers unterliegen könne.

Der siebte Abschnitt fragt in umgekehrter Perspektive nach den Möglichkeiten des Volksgesetzgebers, auf Gerichte einzuwirken, die aus Sicht der Bürger nicht „responsiv“ genug agieren (S. 189-215). Interessant ist hier zunächst die Feststellung, daß bereits die ,progressive“ Bewegung um die Wende vom neunzehnten zum zwanzigsten Jahrhundert mit Klagen über ,judicial activism“ einherging. Miller legt überzeugend dar, daß die Bürger in Staaten mit der Option direktdemokratischer Verfassungsänderung über weit stärkere Korrekturmöglichkeiten verfügen als in solchen, die lediglich den „Recall“ oder die schlichte Nichtwiederwahl von Richtern kennen. Der zeitweise u.a. von Theodore Roosevelt verfochtene Versuch, eine direktdemokratische Verwerfung einzelner Entscheidungen einzuführen, wirkt demgegenüber eher bizarr (S. 193 ff.).

Millers Schlußfolgerung (S. 216-224) besticht einmal mehr durch ihre Abgewogenheit, treibt diese aber vielleicht einen Schritt zu weit. Den Konflikt zwischen Gerichten und direktdemokratischen Initiativen wertet er auf der einen Seite als List der Geschichte: Das System der „,checks and balances“ der Gründungsväter funktioniere, weil die Gerichte die direkte Demokratie im Zaum hielten. Zugleich habe diese das Repräsentativsystem merklich geschwächt, was mit einer Machtverlagerung auf die Gerichte und zugleich mit deren Politisierung einhergegangen sei. Als Remedur empfiehlt Miller den Gerichten Zurückhaltung (S. 223; dies klingt etwas matt, auch wenn der Autor konkrete Kritik am kalifornischen Obersten Gericht übt) sowie eine Einschränkung der verfassungsändernden Initiative; sein Petitum, daß diese sich merklich von der Initiative zur Änderung einfacher Gesetze unterscheiden müsse, verdient uneingeschränkte $\mathrm{Zu}$ stimmung.

Das Werk wird abgerundet durch eine Übersicht zum gerichtlichen „Schicksal"“ direktdemokratischer Initiativen (S. 225-244), ein Literaturverzeichnis sowie einen hilfreichen Index. Es ist jedem dringend zu empfehlen, der entweder an der Entwicklung der direkten Demokratie in den Vereinigten Staaten interessiert ist 
oder rechtsvergleichend informierte Antworten auf die Frage sucht, wie die sachunmittelbare Demokratie auszugestalten ist, um ihre Stärken zu entfalten und ihre Schwächen nicht zum Tragen kommen zu lassen.

Fabian Wittreck

Ulrike Rösler: Das imperfekte Gesetzesinitiativrecht des Volkes. Verlag Dr. Kovač: Hamburg 2010 (Verfassungsrecht in Forschung und Praxis, Bd. 78), 363 S., 98 Euro.

$\mathrm{Zu}$ den plebiszitären Elementen, die unsere repräsentative Demokratie „vergüten" sollen, gibt es sowohl in den Landesverfassungen als auch in der Literatur eine nahezu babylonische Begriffsverwirrung. Verf. unternimmt es daher zu Beginn ihrer Arbeit, einer an der Universität Bremen von Uli Rühl betreuten Dissertation, die verschiedenen Instrumente der direkten Demokratie zu definieren und voneinander abzugrenzen. Sie unterscheidet bei den Initiativrechten des Volkes im Bereich der Gesetzgebung zutreffend zwischen dem ,,imperfekten Gesetzesinitiativrecht“ und der ,,perfekten Initiative als Teil des Volksgesetzgebungsverfahrens“ (S. 5 ff., 45 f.) und grenzt diese Initiativrechte dogmatisch in überzeugender Weise von Petitionen ab (S. 24 ff.). Selbst Massenpetitionen mit Gesetzesvorschlägen vermögen es im Gegensatz zu Gesetzesinitiativrechten nicht, förmliche Gesetzgebungsverfahren in Gang zu setzen, sondern bestenfalls Gesetzesinitiativberechtigte anzuregen, eine Gesetzesinitiative im Sinne der Petenten zu ergreifen (S. 45).

Zentraler Gegenstand der Untersuchung ist das ,imperfekte Gesetzesinitiativrecht des Volkes“, das sich - in Anlehnung an die Terminologie von Pestalozza - von dem perfekten Initiativrecht ,wesentlich dadurch unterscheidet, dass ersteres vom Parlament endgültig zu Fall gebracht werden kann, wohingegen diese Macht bei der perfekten Initiative dem Gesamtvolk vorbehalten ist" (S. 46). Das imperfekte Gesetzesinitiativrecht ist der Sache nach inzwischen in zwölf Landesverfassungen verankert und firmiert dort überwiegend unter dem Begriff „Volksinitiative“.

Der Schwerpunkt der Untersuchung gilt der Zulässigkeit imperfekter Gesetzesinitiativrechte des Volkes zu Verfassungsfragen (,,imperfekte Verfassungsinitiative") und zu den Staatsfinanzen (,imperfekte Finanzinitiative“). Dabei behandelt Verf. allerdings auch die Zulässigkeit von Volksentscheiden speziell in Finanzfragen, um aus deren Zulässigkeit - argumentum a maiore ad minus - auf die Zulässigkeit der nur imperfekten Gesetzesinitiative rückschließen zu können (S. 287). 
Die Untersuchungen zu den „Finanztabus“ in den Landesverfassungen beziehen sich thematisch auf Plebiszite zu Haushaltsgesetzen und -plänen, zu Abgaben, Besoldungs-, Dienst- und Versorgungsbezügen sowie zu finanzwirksamen Gesetzen. Verf. kommt dabei - zumeist gegen die h.M. argumentierend - im Wesentlichen zu folgenden Ergebnissen:

- Soweit Landesverfassungen Finanzinitiativen in Form von Volksbegehren oder Volksinitiativen nicht explizit ausschlössen, gefährdeten diese ,weder die Sachgerechtigkeit von Finanzentscheidungen, noch das parlamentarische Budgetrecht, noch die Leistungsfähigkeit des Staates und begünstigen auch keinen Missbrauch durch bestimmte Interessengruppen“ (S. 331).

- Mit imperfekten Finanzinitiativen könnten auch Vorschläge zum Haushaltsgesetz eingebracht werden, da es insoweit kein Initiativmonopol der Regierung gebe; zulässig seien auch Initiativen zum Abgaben- und Besoldungsrecht (S. $218 \mathrm{ff}$.).

- Selbst erheblich finanzwirksame Gesetze könnten im Wege der Volksgesetzgebung beschlossen werden; damit seien entsprechende imperfekte Gesetzesinitiativrechte des Volkes erst recht zulässig (S. 283).

Die oft kühnen Attacken der Verf. gegen die h.M. wirken manches Mal verbal etwas überheblich: „schlicht unzutreffend“ (S. 275), „Schlag in die Luft“ (S. 260), „gänzlich fehlgeleitet“ (Fn. 1070). Sie verneint z.B. das Monopol der Regierung zur Einbringung von Gesetzesentwürfen zum Haushalt oder hält eine Volksgesetzgebung mit selbst erheblichen finanziellen Auswirkungen für zulässig; das mag de lege lata jedoch durchaus zweifelhaft sein. Wenn sie sich dabei allerdings engagiert gegen Stimmen innerhalb der h.M. wendet, die dem Volk ausdrücklich oder unausgesprochen bei dessen Finanzinitiativen eigennützige Motive, fehlendes Gemeinwohlbewusstsein, mangelnden Sachverstand und intellektuelle Überforderung unterstellen und damit das Volk eher als Gefahr denn als sinnvolles Korrektiv neben den Parlamenten ansehen, kann man ihr nur beipflichten. Zum einen liegt in dieser Geringschätzung des Volkes ein Wertungswiderspruch zu seiner verfassungsrechtlichen Befugnis, selbst über komplizierteste Gesetzesmaterien entscheiden zu dürfen; zum anderen ist zu fragen, wer denn die katastrophale Überschuldung unserer öffentlichen Haushalte zu verantworten hat, doch nicht das Volk, sondern die Parlamente als Gesetzgeber (vgl. dazu zutreffend S. Jutzi, NJ 2001, S. 646; BgbVerfG LKV 2002, S. 80). Jede Mark bzw. jeder Euro, der zusätzlich in die öffentlichen Kassen kam, wurde gefördert von einer großen Portion Eigennutz der Berufsabgeordneten - beileibe nicht zur Haushaltskonsolidierung genutzt, sondern für neue Wohltaten verwendet. Wurde die verfassungsrechtlich verpflichtende Schuldenbremse nicht gerade deswegen in das Grundgesetz eingefügt, weil Abgeordnete selbst nicht mehr an 
ihre eigene politische Vernunft und Durchsetzungsfähigkeit glaubten und sich daher, die eigene Schwäche erkennend, diese verfassungsrechtliche Kandare anlegten? Sind die Bürger demgegenüber nicht vielleicht doch die besseren Haushälter, worauf auch ausländische Erfahrungen hindeuten?

Verf. macht es sich allerdings bei der Frage, ob gewisse Finanzvorbehalte zur Sicherung der Leistungsfähigkeit des Staates bei einer finanzwirksamen Volksgesetzgebung erforderlich sind, dann doch etwas zu leicht, wenn sie darin keine „,nennenswerte Gefahr“ sehen will (S. 268), zumal die Kostenrelevanz in der öffentlich plebiszitären Meinungsbildung Beachtung fände. Außerdem argumentiert sie, dass den Parlamenten ja immer noch die Möglichkeit bleibe, ein volksbeschlossenes Gesetz, das den Staat in existenzielle Nöte bringt, „kurzer Hand“ abzuändern oder aufzuheben (S. 268).

Man kann jedoch nicht vollmundig Fähigkeiten des mündigen Bürgers preisen (S. 257) und gleichzeitig das Parlament als haushaltsrechtlichen Rettungsanker gegenüber plebiszitärer Unvernunft ins Feld führen.

Die geltende Verfassungsrechtslage, wonach der Volksgesetzgeber im Gegensatz zum parlamentarischen Gesetzgeber nicht dem haushaltsrechtlichen Deckungsvorbehalt unterliegt (anders nur in Niedersachsen, Art. 68 Abs. 1 NdsVerf.), d.h. finanzrelevante Gesetze in keiner Weise mit einem Kostendeckungsvorschlag versehen sein müssen, erscheint zumindest verfassungspolitisch äußerst problematisch. Das Volk kann m.E. nicht von der Kostenverantwortung für ,,seine" Gesetze freigestellt werden. Zu erwägen ist allerdings, ob es bei der Erarbeitung der eigenen Deckungsvorschläge Informationen und sonstige Unterstützung von der Regierung erhalten muss und sich dabei auf wesentliche Eckpunkte beschränken darf.

Diese rechtspolitischen Anmerkungen leiten über zu dem Hinweis, dass sich Verf. nicht nur mit ihrem Thema de lege lata, sondern erfreulicherweise auch rechtspolitisch befasst. Derzeit ist die Einführung einer Volksgesetzgebung auf Bundesebene, die wegen der dazu erforderlichen Ergänzung des Grundgesetzes einer Zweidrittelmehrheit in Bundestag und Bundesrat bedarf, politisch nicht durchsetzbar. Im Koalitionsvertrag von CDU/CSU und FDP ist daher nur von einer vorsichtigen Weiterentwicklung des Petitionsrechts die Rede. Danach sollen bei „Massenpetitionen“ „über das im Petitionsausschuss bestehende Anhörungsrecht hinaus eine Behandlung des Anliegens im Plenum des Deutschen Bundestages unter Beteiligung der zuständigen Ausschüsse vorgesehen“ werden. Verf. plädiert daher als Kompromiss für die Einführung einer imperfekten Gesetzesinitiative des Volkes ohne jeden Themenausschluss in das Grundgesetz, für dessen wesentliche Ausgestaltung sie konkrete Detailvorschläge unterbreitet. 
In einem abschließenden Kapitel widmet sich Verf. ganz aktuell der mit Art. 11 Abs. 4 EUV bzw. Art. 24 Abs. 1 AEUV eingeführten europäischen Bürgerinitiative.

Verf. bereichert mit ihrer Arbeit nicht nur die laufenden Debatten um die Auslegung des geltenden Verfassungsrechts zu den plebiszitären Beteiligungsrechten des Volkes, mit Vorrang des imperfekten Gesetzesinitiativrechts, sondern auch die allgemeine rechtspolitische Diskussion um die unmittelbare Demokratie, die aufgrund des zunehmenden Vertrauensverlustes der Bürger in ihre demokratischen Repräsentanten in den Parlamenten (vgl. J. Linck, Zurück zum ehrenamtlichen Landesparlamentarier? in: H.H. v. Arnim [Hrsg.], Defizite in Staat und Verwaltung, 2010, S. 92 f.) immer größere Bedeutung und Brisanz erhält.

Joachim Linck 